\title{
Using repetitive Transcranial Magnetic Stimulation (rTMS) Method in the Treatment of Major Depressive Disorder (MDD)
}

Behzad Saberi

Medical Research, Esfahan, Iran

*Corresponding author: Behzad Saberi, Medical Research, Esfahan, Iran.

Received date: August 07, 2020; Accepted date: August 31, 2020; published date: September 21, 2020

Citation: Behzad Saberi. Using repetitive Transcranial Magnetic Stimulation (rTMS) Method in the Treatment of Major Depressive Disorder (MDD. J Clinical Research and Reports, 5(4); DOI:10.31579/2690-1919/116

Copyright: $\odot 2020$ Behzad Saberi. This is an open access article distributed under the Creative Commons Attribution License, which permits unrestricted use, distribution, and reproduction in any medium, provided the original work is properly cited.

\section{Abstract :}

Repetitive Transcranial Magnetic Stimulation (rTMS) as a noninvasive method of brain stimulation, has shown various diagnostic and therapeutic potentials in neurologic and psychiatric disorders.

Major Depressive Disorder (MDD) as one of the global challenges in mental health disorders, requires new therapeutic strategies to be developed to be treated in addition to the usual therapeutic strategies. Repetitive Transcranial Magnetic Stimulation or rTMS is one of the new treatment methods for the Major Depressive Disorder which is promising new developments in the treatment of MDD. rTMS is approved by the Food and Drug Administration to treat resistant depression in 2008. According to the recent guidelines in the Europe and North America, rTMS can be considered the first line treatment strategy for the MDD.

This is a brief review study on the effects of using repetitive Transcranial Magnetic Stimulation in the treatment of Major Depressive Disorder in clinical practice.

It is important for the mental health care professionals who deal with the patients with the Major Depressive Disorder during clinical practice, to be aware of the repetitive Transcranial Magnetic Stimulation method as an option to treat MDD specifically in the cases who do not respond well to usual therapeutic strategies or whom encounter with unfavorable drug treatment side effects which need the treatment strategies to be changed.

Key words: Repetitive Transcranial Magnetic Stimulation (rTMS) - Major Depressive Disorder (MDD)

\section{Body:}

Repetitive Transcranial Magnetic Stimulation or rTMS is done by placing an electronic coil on the scalp. By doing this, different parts of the brain would receive short magnetic pulses in a repetitive manner. Dorsolateral Prefrontal Cortex or DLPFC is the most prominent area of the brain which would be the target of the repetitive Transcranial Magnetic Stimulation method in the treatment of the Major Depressive Disorder. Dorsolateral Prefrontal Cortex has been found to be hypoactive during depression. So some processes like attention, decision-making and working memory which have been found to be regulated by the Dorsolateral Prefrontal Cortex region of the brain, would be affected by the Major Depressive Disorder.

Repetitive transcranial magnetic stimulation employment in the treatment of resistant and also mild forms of depression is approved by the Food and Drug Administration in 2008.

Repetitive Transcranial Magnetic Stimulation sessions would be done for several days a week and for a time period which lasts about six weeks and each session's time would last for twenty to forty minutes. The efficacy of employing the repetitive Transcranial Magnetic Stimulation method in the treatment of Major Depressive Disorder and various types of depressive disorders, is variable according to different research studies.

Using the "Slow" form of the repetitive Transcranial Magnetic Stimulation method would be more effective in comparison with the "Fast" form of that. Also employing the Slow form of the repetitive Transcranial Magnetic Stimulation method can be safely used with higher intensities during treatment.

In some studies, combination therapy with repetitive Transcranial Magnetic Stimulation method and Antidepressants, has been demonstrated to have additive therapeutic effects in the treatment of Major Depressive Disorder.

In some of the other studies, using repetitive Transcranial Magnetic Stimulation method in comparison with Electroconvulsive Therapy or ECT, has been demonstrated to have similar therapeutic effects. Also the results of some of the other different studies, have shown that using repetitive Transcranial Magnetic Stimulation method for three to four weeks to treat Major Depressive Disorder, would be safe and does not have any side effects. 
Although not all the studies have supported using repetitive Transcranial Magnetic Stimulation method to treat Major Depressive Disorder, but most of the studies have supported the effectiveness of using the repetitive Transcranial Magnetic Stimulation method in the treatment of Major Depressive Disorder by employing it alone or in combination with other treatment strategies like Pharmacological ones.

\section{Conclusion:}

Repetitive Transcranial Magnetic Stimulation can be an effective treatment method for Major Depressive Disorder and some other depressive pathologies. Additional research studies should be done in evaluating the effectiveness of using repetitive Transcranial Magnetic Stimulation in the treatment of clinical symptoms of the Major Depressive Disorder and other depressive pathologies. It is important for the clinicians who are dealing with the patients suffering from various forms of depressive pathologies including Major Depressive Disorder, to have knowledge about repetitive Transcranial Magnetic Stimulation method as an important and effective therapeutic strategy in clinical practice.

\section{References}

1. Berlim MT, van den Eynde F, Tovar-Perdomo S, et al. Response, remission and drop-out rates following highfrequency repetitive transcranial magnetic stimulation (rTMS) for treating major depression: a systematic review and metaanalysis of randomized, double-blind and sham-controlled trials. Psychol Med. 2014;44(2):225-239.

2. Perera T, George MS, Grammer G, et al. The Clinical TMS Society consensus review and treatment recommendations for TMS therapy for major depressive disorder. Brain Stimulat. 2016;9(3):336-346.
3. Sackeim HA. Acute continuation and maintenance treatment of major depressive episodes with transcranial magnetic stimulation. Brain Stimulat. 2016;9(3):313-319.

4. Hutton TM. The clinical application of transcranial magnetic stimulation. Psychiatr Ann. 2014;44(6):305-309.

5. George MS, Wassermann EM, Williams WA, et al. Daily repetitive transcranial magnetic stimulation (rTMS) improves mood in depression. Neuroreport. 1995;6(14):1853-1856.

6. Wassermann EM, Zimmermann T. Transcranial magnetic brain stimulation: therapeutic promises and scientific gaps. Pharmacol Ther. 2012;133(1):98-107.

7. McGirr A, Van den Eynde F, Tovar-Perdomo $\mathrm{S}$, et al. Effectiveness and acceptability of accelerated repetitive transcranial magnetic stimulation (rTMS) for treatmentresistant major depressive disorder: an open label trial. J Affect Disord. 2015;173(1):216-220.

8. Lefaucheur JP, André-Obadia N, Antal A, et al. Evidence-based guidelines on the therapeutic use of repetitive transcranial magnetic stimulation (rTMS) Clin Neurophysiol. 2014;125(11):2150-2206.

9. Kellner CH, Knapp RG, Petrides G, et al. Continuation electroconvulsive therapy vs pharmacotherapy for relapse prevention in major depression: a multisite study from the Consortium for Research in Electroconvulsive Therapy (CORE) Arch Gen Psychiatry. 2006;63(12):1337-1344. 